\title{
On Enrichment Culturing and Transferring Technique
}

\author{
Ji-Dong Gu \\ Environmental Science and Engineering Program, Guangdong Technion - Israel Institute of Technology, 241 Daxue Road, Shantou, \\ Guangdong 515063, China
}

\begin{abstract}
Enrichment culturing and transferring technique is frequently used to obtain specific functional microorganisms for more in-depth investigations from the complex samples containing a wide range of different microorganisms. This technique is fundamental and critically important in research of microbial ecology, environmental microbiology, and environmental science, but the proper practice of this technique in applications remains ambiguous to some investigators. Because of this situation, misuse and lack of comprehensive understanding of the meaning of this technique are frequently found in manuscripts or even publications. This article provides a discussion about this technique and the meaning for practical use to enhance research for high-quality results and the scientific information effectively when used. The key elements of this technique include cultural medium composition, culturing and incubation, frequency of transferring for enrichment, procedures prior to the isolation and purification to obtain possibly pure cultures or enrichments of the capable microorganisms for further biochemistry and physiology investigations. The information is intended to improve the basic understanding of this technique for a more effective and efficient applications in research to advance the basic and fundamental information and to pave the way for more innovative research and discoveries to science.
\end{abstract}

Keywords: Enrichment and transfer technique, isolation, pollutant metabolism, degradation pathway

Correspondence to: Ji-Dong Gu, Environmental Science and Engineering Program, Guangdong Technion - Israel Institute of Technology, 241 Daxue Road, Shantou, Guangdong 515063, China; E-mail: jidong.gu@gtiit.edu.cn

Received: March 19, 2021; Accepted: April 7, 2021; Published Online: April 9, 2021.

Citation: Ji-Dong Gu, 2021. On enrichment culturing and transferring technique Applied Environmental Biotechnology, 6(1): 1-5. http://doi.org/10.26789/AEB.2021.01.001

Copyright: On enrichment culturing and transferring technique. () $2021 \mathrm{Ji}$-Dong Gu. This is an Open Access article published by Urban Development Scientific Publishing Company. It is distributed under the terms of the Creative Commons Attribution-Noncommercial 4.0 International License, permitting all non-commercial use, distribution, and reproduction in any medium, provided the original work is properly cited and acknowledged.

\section{Introduction}

Microbial transformation and degradation of toxic and industrial chemicals are important research information and also for biotechnological applications in cleaning up the polluted sites (Alexander, 1999; Gu, 2018; Schwarzenbach et al., 2006; Zylstra and Kukor, 2005). To achieve the goals of both any research and applications, isolation of the specific microorganisms are often the first step required prior to the establishment and substantiation of the biochemical transformation processes, the enzymes and genes involved, in situ expression and activity of the microorganisms, and applications in cleaning up contaminated sites (Gu, 2016; 2020a; 2020b). Traditionally, pure cultures of microorganisms are the basic requirements to establish the taxonomy and classification as well as the physiology and biochemistry for a comprehensive documentation of the relevant information in microbiology (Alexander, 1999).

Current laboratory methods on culturing and isolation of microorganisms can only achieve for no more than $1 \%$ of the natural population using the currently available laboratory culturing techniques (Amann et al., 1995). It is still a great difficult task to isolate or enrich specific functional and new microorganisms from complex environmental samples of soils, sediments, and sludge before the further biochemical and physiological analyses of the pure culture of any new microorganisms. One of the reasons to the current situation is inherited mainly from the commercially cultural media available and also the established agar plates for isolating techniques initially used for medical microbiology. For example, the commercial microbiological media contain high concentrations of nutrients that are far greater than those found in the natural ecosystems by at least thousands to millions of orders. In such case, the fast growing microorganisms capable of utilizing and survival at such extremely high concentrations of nutrients (osmotic pressure and substrate sequestration through transporters) are isolated easily. This approach and strategy has been very effective in isolation of medically important pathogens and opportunistic ones due to the enrichment of them before disease development and symptom, but microbial ecology of the natural ecosystems or polluted environments are different and shall be dealt with using different technical methods because of the oligotrophic condition and toxicity, respectively. Though less emphasized, this technique of enrichment is a foundation and basis for high impact research for new discoveries as illustrated with ammonia-oxidizing archaea (AOA) (Könneke et al., 2022b), Anammox (Mulder et al., 1995), n-damo (Raghoebarsing et al., 2006), Comammox (Daims, 2015; 2020c) for the past almost 30 years as examples. This is also true in environ- 
mental microbiology when degradation of toxic pollutants is the research objective, but the toxicants as both substrate for microbial growth and also toxic chemical must be balanced for the identification of the concentration range feasible for enrichment culturing. Many degradation studies of pollutants frequently mention the use of enrichment culturing technique in the research and results, but the meaningful application and an adequate understanding of the technique in practice are apparently lack or weak in many of them on microbial transformation (Ma et al., 2020; Gu, 2016). The challenges with non-visible microorganisms, fungi, bacteria, and archaea are apparently their smaller sizes than animals and plants, and sophisticated instrumentation or analytical techniques are required to detect the selective one from the general population.

\section{Enrichment culturing and Transfer}

\subsection{Culture medium composition}

The purposes of establish any enrichment are to increase the targeted population of microorganisms by a combination of means, selective substrate, medium composition, culturing conditions, and frequent transfer. Culture medium composition for microorganisms is fundamentally important to the success of enrichment and culturing. To isolate microorganism capable of degrading any selective organic pollutant, the organic chemical of concern shall be the sole source of carbon and energy in the medium for heterotrophic microorganisms, but another carbon source is included in the cultural medium in many studies to allow easy positive results on microorganisms (Ma et al., 2020; Zhang et al., 2021). As a result, the proliferation of microorganisms in the culture medium cannot be those responsible for utilizing the organic environmental pollutants. The purpose of enrichment by selection pressure by the amended organic chemical is forgotten completely in practice and the results introduce bias on claim that the isolated microorganism utilizes the target chemical for growth because co-metabolism is the mechanism involved for the transformation of the target chemical in reality (Horvath, 1972; Hulbert and Krawiec, 1977).

A specific chemical of choice shall be a source of carbon and energy for the microorganisms in the enrichment culture to utilize it for population growth when the chosen concentration is obviously below the toxicity level (Gu, 2020b). For many environmental pollutants, water solubility of them is a major limiting factor to allow high concentration achieved in cultural media (Ji et al.,2020a; 2020b; Liu et al., 2020a; 2020b; Zhou et al., 2019), and, as a result, bioaccumulation in organisms and animals, and biomagnification through the food chain transfer are often observed ( $\mathrm{Gu}, 2019)$. The specific concentration used in cultural media is far exceeding the solubility of them in water in many publications (Ma et al., 2020; Zhang et al., 2021) and the determination of concen-

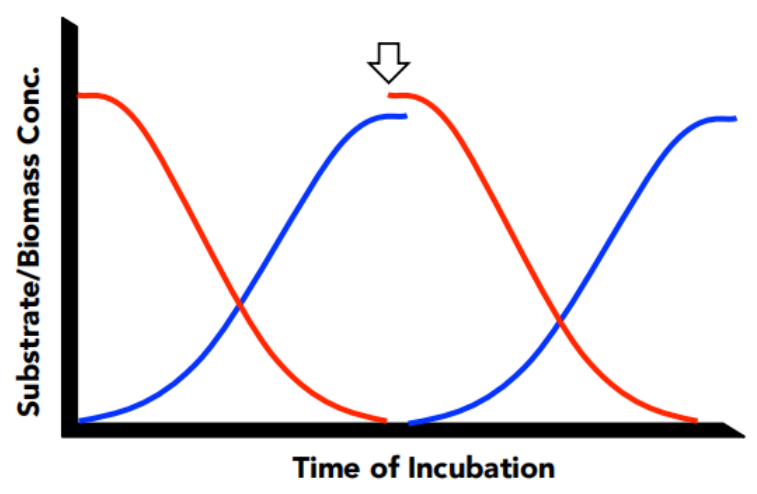

Figure 1. An illustration of substrate (in red) depletion and at the same time population increase (in blue) during culturing in the initial enrichment culture and then first enrichment transfer culture, showing similar patterns for substrate and microbial population.

tration during incubation becomes less reliable or inaccurate to show the indicative degradation as claimed. This factor has not been addressed carefully in a large number of the publications in environmental science and engineering.

\subsection{When to transfer}

When a microorganism is capable of metabolizing a specific chemical, its population and also the other microorganisms from the initial inoculum cannot be distinguished by conventional monitoring techniques, plate counting or spectrophotometry. However, the substrate concentration dynamics can provide an indication on the population utilizing the substrate potentially because these two are generally inversely related (Figure 1). This becomes more convincing and evident after a number of enrichment transfers being made and similar results can be repeatedly observed (Gu and Berry, 1991; 1992). After an appreciable quantities of the substrate have been disappeared from the culture medium, e.g., 60-70\%, the first enrichment transfer can be made to eliminate the mixed population of microorganisms from the inoculum and also to further enhance the capability of the metabolizing microorganism to further increase its population and competitiveness in the culture medium ( $\mathrm{Gu}$ and Berry, 1991; 1992). Many published papers stop at the initial enrichment without any transferring, which does not carry the meaning of enrichment and transfer because no elimination of the non-wanted microorganisms has been made by any attempt and the culture still contains a mixture of far too many non-degraders than the targeted few. Others make the first transfer and then plate the culture medium directly onto nutrient rich agar plates to isolate microorganisms. Both of them conduct the so-called 'enrichment and transfer' only partially or preliminarily and the results are not effective to allow selecting any truly degrading microorganisms by the transferring steps to eliminate. In conducting the procedure correctly, a number of transfers shall be made to exhaust the microbial contaminants and also confirm the biochemical capability of the possible microbial 
isolates by detecting the substrate concentration repeatedly (Figure 2). By observing the transfers, the enriched microorganisms are competitive in utilizing the substrate as the sole source of carbon and energy and also provide high chance of purifying it with the selected substrate in the agar plates. The foundation and logic of enrichment and transfer must be understood well enough to serve the research purpose for better achievements.

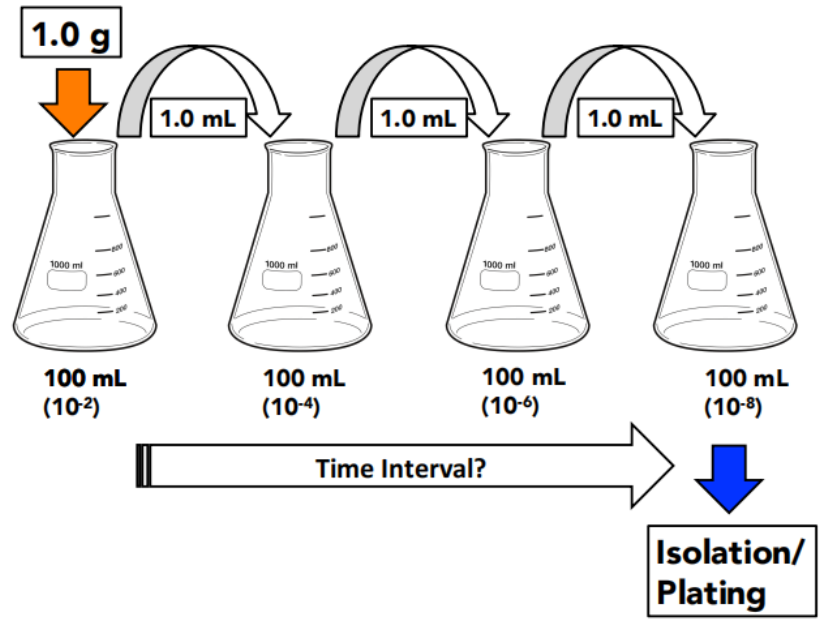

Figure 2. A schematic illustration of the enrichment culturing prior to isolation and identification of culturable microorganisms for capability of degrading selective environmental organic toxicant

\subsection{Enrichment processes}

Enrichment transfer processes play a very important role in enriching the degrading microorganisms while eliminating those that do not participate in degradation processes or contribute less significantly. This procedure can be illustrated in Figure 2, in which a starting inoculum of $1.0 \mathrm{~g}$ of inoculum materials, namely soil or sludge or water sample can be used as the source of microorganisms. The organic carbon in the initial culture is $1 \%$ ( 1 in $100 \mathrm{ml}$, total volume used) and, when enrichment transfers are being made, the first enrichment ( $2^{\text {nd }}$ flask in Figure 2) and subsequent ones contain $100 \mathrm{ppm}, 1 \mathrm{ppm}$ and $10 \mathrm{ppb}$ of the initial carbon from the inoculum as carried over in subsequent enrichment transfer cultures. Since the target chemical is used at a relatively constant concentration in each enrichment transfers, this allows the enrichment transfers to firmly establish that the selected microorganisms showing a population growth use the candidate substrate effectively, not the carried over from the inoculum to support the biomass or interfere with the observed results (Figure 2). This series of transferring steps is mostly missing from many investigations unfortunately (Ma et al., 2020; Zhang et al., 2021). This shall be a major emphasis on educating the young researchers to full grasp the meaning of the enrichment and transferring technique to better conduct their research efficient and scientifically to serve the science.

\subsection{Frequency of transferring}

Given the importance of the enrichment transfers, what shall be the time interval to make a transfer or next one? Obviously, this depends on the substrate and its concentration, and also the growth rate of the candidate microorganisms, which are difficult to monitoring specifically due to the complex nature of the enrichment culture with so many different microorganisms at the same time. However, it is relatively easier to monitoring the substrate concentration depletion during the incubation as an indicator for increase of the whole microbial population (Figure 1). If this is used, further enrichment transfers may result in positive growth of a certain and more and more selective microbial population continuously in the successive enrichment transfers. This is the sole reason for transferring in practice. But, it is also expected that a delayed concentration change or no change at all may be observed which suggest that the anticipated microorganisms have not been successfully enriched between the transfer progression, possibly slower growth rate and other factors involved. At this point, a new revised plan shall be considered to allow the research objective met hopefully through reasoning and logical laboratory efforts. The other reasons may be possibly due to lack of growth cofactors or other specific nutritional requirements for the candidate microorganisms or simply the environmental conditions in the culture medium after further transfers are being made. This situation has been experienced with many of the dehalogenating microorganisms reported before (Maymo-Gatell et al., 1997; Shelton and Tiedje, 1984). Unfortunately, there is no available textbook instructions or guidance on the specific steps taken to identify the causes or solve this issue productively, but trial-and-error is essential to find out the solution logically and innovatively to advance the research for a big breakthrough. The challenges involved here also mean great opportunities ahead as evidenced by the major breakthroughs made (Maymo-Gatell et al., 1997; Shelton and Tiedje, 1984).

\subsection{Others to be considered}

In the marathon for enriching the target microorganisms, time interval for the enrichment transfer is an important factor because it shall allow the multiplication of the candidate microorganisms more than the non-targeted ones to make transferring meaningful to benefit the enrichment and isolation. This factor can be balanced between the growth rate of the target microorganisms and elimination of the co-existed population to achieve the best results efficiently. Because of chemical composition in the medium and incubation conditions, including osmotic pressure, cations and anions available, $\mathrm{pH}$ value and temperature, aeration and dissolved oxygen or agitation of the cultural medium, the targeted microorganisms may respond to these or a selective one or a few sensitively. For example, Anammox and n-damo bacteria grow best under low dissolved oxygen in chemostat fermenters (Mulder et al., 1995; Raghoebarsing et al., 2006), but not strictly 
anaerobic conditions without any oxygen while AOA and Comammox require aerobic condition but no agitation of the culture medium during incubation (Könneke et al., 2022b; Daims, 2015). These requirements are not found in any laboratory manual or textbook previously, and they are first hand experiences from the pioneering researchers conducting the enrichment culturing and observing meticulously for any changes at each small step to accumulate an overall understanding. The greater challenges are obvious to everyone in conducting original and innovative research, but the outcome as a reward is also significant greater worthwhile the efforts put into this. Currently available genomics and transcriptomics coupling with chemistry also allow a peek into the microbial world for the rich diversity of microorganisms and biochemical capability (Chen, 2020; Gu, 2021; Liu et al., 2018; Liu et al., 2020a; 2020b; Yang et al., 2020a; 2020b; 2020c).

\section{Future Perspectives}

With the rich repertoire of new microorganisms unknown in pure cultures or enrichment cultures of natural and impacted environments, research protocols on enrichment and isolation of different functional groups of microorganisms need to be further improved and refined to advance our technical capabilities in manipulation environmental samples for high success rate in isolating and purifying new microorganisms from the ecosystems. The success from such effort allows more detailed research on the biochemistry and physiology of the new microorganisms to advance our knowledge on the rich diversity of microbial metabolism and enrich the phylogenetic and evolutionary understanding of them to the current available tree of life for a better knowledge in life science and biology.

It is a reality that no standard methods are available in this frontier research in microbiology and the existing ones can serve as references, but more new protocols or methods can be developed from more successful cases. Because of this, the methods used current and reported in publications must be reliable and also on a solid foundation for further microbiological research development. A lack of such requirement and standards in quality control would delay the advancement of microbial ecology, especially on isolation and enrichment of new functional microorganisms from the diverse and anthropogenic impact ecosystems.

\section{Acknowledgements}

Research in this laboratory is supported by National Natural Science Foundation of China.

\section{Conflict of Interest}

Author declares that there is no conflict of interest in the information presented here.

\section{Ethical approval}

This article does not contain any studies with human participants or animals performed by the author involved.

\section{References}

Alexander, M., 1999. Biodegradation and Bioremediation ( $2^{\text {nd }}$ ed.). Academic Press, San Diego, California.

Amann, R.I., W. Lidwig, K.-H. Schleifer, 1995. Phylogenetic identification and in situ detection of individual microbial cells without cultivation. Microbiological Reviews, 59: 143-169.

Chen, J., L. Zhou, Y.-F. Liu, Z.W. Hou, W. Li, S.M. Mbadinga, J. Zhou, T. Yang, J.-F. Liu, S.-Z. Yang, X. L. Wu, J.-D. Gu, B.-Z. Mu, 2020 Synthesis and mass spectra of rearrangement bio-signature metabolites of anaerobic alkane degradation via fumarate addition. Analytical Biochemistry, 600: 1137-1146. http://doi.org/10.1016/j.ab.2020.113746

Daims, H., E.V. Lebedeva, P. Pjevac, P. Han, C. Herbold, M. Albertsen, N. Jehmlich, M. Palatinszky, J. Vierheilig, A. Bulaev, R.H. Kirkegaard, M. von Bergen, T. Rattei, B. Bendinger, P.H. Nielsen, M. Wagner, 2015. Complete nitrification by Nitrospira bacteria. Nature, 528: 504-509. http://doi.org/10.1038/nature16461

Gu, J.-D., 2016. Biodegradation testing: so many tests but very little new innovation. Applied Environmental Biotechnology, 1(1): 92-95. http://doi.org/10.26789/AEB.2016.01.007

Gu, J.-D., 2018. Bioremediation of toxic metals and metalloids for cleaning up from soils and sediments. Applied Environmental Biotechnology, 3 (2): 48-51. http://dx.doi.org/10.26789/AEB.2018.02.006

Gu, J.-D., 2019. Microbial ecotoxicology as an emerging research subject. Applied Environmental Biotechnology, 4 (1): 1-4. http://doi.org/10.26789/AEB.2019.01.001

Gu, J.-D., 2020a. Anthroposphere, a new physical dimension of the ecosystems. Applied Environmental Biotechnology, 5 (1): 1-3. http://doi.org/10.26789/AEB.2020.01.001

Gu, J.-D., 2020b. On environmental biotechnology of bioremediation. Applied Environmental Biotechnology, 5 (2): 3-8. http://doi.org/10.26789/AEB.2020.02.002

$\mathrm{Gu}$, J.-D., 2021. Biodegradability of plastics: the issues, recent advances and future perspectives. Environmental Science and Pollution Research, 28 (2): 1278-1282. http://doi.org/10.1007/s11356-020-11501-9

Gu, J.-D., D.F. Berry, 1991. Degradation of substituted indoles by an indoledegrading methanogenic consortium. Applied and Environmental Microbiology, 57: 2622-2627. http://doi.org/10.1007/BF01575855

$\mathrm{Gu}$, J.-D., D.F. Berry, 1992. Metabolism of 3-methylindole by a methanogenic consortium. Applied and Environmental Microbiology, 58: 2667-2669. http://doi.org/10.1007/BF00584467

Horvath, R.S., 1972. Microbial Co-metabolism and the degradation of organic compounds in nature. Microbiological Reviews, 36: 146-155. http://doi.org/10.1128/MMBR.36.2.146-155.1972

Hulbert, M.H., S. Krawiec, 1977. Cometabolism: A critique. Journal of Theoretical Biology, 69: 287-291. http://doi.org/10.1016/0022-5193(77)90137-0

Ji, J.-H., Y.-F. Liu, L. Zhou, M. Irfan, S.M. Mbadinga, P. Pan, J. Chen, J.-F. Liu, S.-Z. Yang, W. Sand, J.-D. Gu, B.-Z. Mu, 2020a. Methanogenic biodegradation of $\mathrm{C}_{13}$ and $\mathrm{C}_{14} n$-alkanes activated by addition to fumarate. International Biodeterioration \& Biodegradation, 153: 10491072 . http://doi.org/10.1016/j.ibiod.2020.104994

Ji, J.-H., L. Zhou, S.M. Mbadinga, M. Irfan, Y.-F. Liu, P. Pan, Z.-Z. Qi, J. Chen, J.-F. Liu, S.-Z. Yang, J.-D. Gu, B.-Z. Mu, 2020b. Methanogenic biodegradation of $\mathrm{C}_{9}$ to $\mathrm{C}_{12} n$-alkanes initiated by Smithella via fumarate addition mechanism. AMB Express, 10 (1): 23. http://doi.org/10.1186/s13568-020-0956-5 
Könneke, M., A.E. Bernhard, R. José, C.B. Walker, J.B. Waterbury, D.A. Stahl, 2005. Isolation of an autotrophic ammonia-oxidizing marine archaeon. Nature, 437: 543-546. http://doi.org/10.1038/nature03911

Liu, X., L. Shi, J.-D. Gu, 2018. Microbial electrocatalysis: Redox mediators responsible for extracellular electron transfer. Biotechnology Advances, 36: $1815-1827$. http://doi.org/10.1016/j.biotechadv.2018.07.001

Liu, Y.-F., J. Chen, Z.-L. Liu, L.-B. Shao, D.-D. Lin, L. Zhou, S.-Z. Yang, J.F. Yang, J.-D. Gu, B.-Z. Mu, 2020a. Anaerobic degradation of paraffins by thermophilic actinobacteria under methanogenic conditions. Environmental Science \& Technology, 54: 10610-10620. http://doi.org/10.1021/acs.est.0c02071

Liu, Y., J. Chen, L. Zaramela, L.-Y. Wang, S.M. Mbdinga, Z.-W. Hou, X.-L. Wu, J.-D. Gu, K. Zengler, B.-Z. Mu, 2020b. Genomic and transcriptomic evidence supports methane metabolism in Archaeglobi. mSystems, 10: 11. http://doi.org/10.1128/mSystems.00651-19

Ma Q., S. Liu, S. Li, J. Hu, M. Tang, Y. Sun, 2020. Removal of malodorant skatole by two enriched microbial consortia: performance, dynamic, function prediction and bacterial isolation. Science of the Total Environment, 725: 138416.

http://doi.org/10.1016/j.scitotenv.2020.138416

Maymo-Gatell, X., Y.-t. Chien, J.M. Gosett, S. Zinder, 1997. Isolation of a bacterium that reductively dechlorinates tetrachloroethane to ethene. Science, 276: 1568-1571.

http://doi.org/10.1126/science.276.5318.1568

Mulder, A., A. van de Graaf, L.A. Robertson, J.G. Kuenen, 1995. Anaerobic ammonium oxidation discovered in a denitrifying fluidized bed reactor. FEMS Microbiology Ecology, 16: 177-183. http://doi.org/10.1111/j.1574-6941.1995.tb00281.x

Raghoebarsing, A.A., A. Pol, K.T. van de Pas-Schoonen, A.J.P. Smolders, K.F. Ettwig, W.I.C. Rijpstra, S. Schouten, J.S.S. Damste, H.J.M. Op den Camp, M.S.M. Jetten, M. Strous, 2006. A microbial consortium couples anaerobic methane oxidation to denitrification. Nature, 440: 918-921. http://doi.org/10.1038/nature04617

Schwarzenbach, R.P., B.I. Escher, K. Fenner, T.B. Hofstetter, C.A. Jonson, U. von Gunten, B. Wehrli, 2006. The challenge of micropollutants in aquatic systems. Science, 313: 1072-1077. http://doi.org/10.1126/science.1127291

Shelton, D.R., J.M., Tiedje, 1984. Isolation and partial characterization of bacteria in an anaerobic consortium that mineralizes 3-chlorobenzoic acid. Applied and Environmental Microbiolology, 48: 840-848. http://doi.org/10.1128/AEM.48.4.840-848.1984

Yang, Y., M. Li, Z. Hu, H. Shim, J.-G. Lin, X. Li, J.-D. Gu, 2020a. Deep insights into the active microbiota in four full-scale wastewater treatment plants showing visible anammox granules by $16 \mathrm{~S}$ rRNA gene and $16 \mathrm{~S}$ rRNA high-throughput sequencing. Journal of Cleaner Production, 276: 124176.

http://doi.org/10.1016/j.jclepro.2020.124176

Yang, Y., J. Pan, Z. Zhou, J. Wu, Y. Liu, J.-G. Lin, Y. Hong, X. Li, M. Li, J.-D. Gu, 2020b. Complex microbial nitrogen-cycling networks in three distinct anammox-inoculated wastewater treatment systems. Water Research, 168: 115142.

http://doi.org/10.1016/j.waters.2019.115142

Yang, Y., H. Daims, Y. Liu, C. Herbold, P. Pjevac, J.-G. Lin, M. Li, J.-D. Gu, 2020c. Activity and metabolic versatility of complete ammonia oxidizers in full-scale wastewater treatment systems. mBio, 11: e 03175-19. http://doi.org/10.1128/mBio.03175-1

Zhang, Z., H. Guo, J. Sun, X. Gong, C. Wang, H. Wang, 2021. Exploration of the biotransformation processes in the biodegradation of phenanthrene by a facultative anaerobe, strain $\mathrm{PheF} 2$, with $\mathrm{Fe}(\mathrm{III})$ or $\mathrm{O} 2$ as an electron acceptor. Science of the Total Environment, 750: 142245. http://doi.org/10.1016/j.scitotenv.2020.142245

Zhou, Z., B. Liang, L.-Y. Wang, J.-F. Liu, B.-Z. Mu, H. Shim, J.-D. Gu, 2019. Identify the core bacterial microbiome of hydrocarbon degradation and a shift of dominant methanogenesis pathways in oil and aqueous phases of petroleum reservoirs with different temperatures from China Biogeosciences, 16: 4229-4241. http://doi.org/10.5194/bg-16-4229-2019

Zylstra, G.J., J.J. Kukor, 2005. What is environmental biotechnology? Current Opinion in Biotechnology, 16(3): 243-245. http://doi.org/10.1016/j.copbio.2005.05.001 